\title{
Automated Demand Response from Home Energy Management System under Dynamic Pricing and Power and Comfort Constraints
}

\author{
Sereen Althaher, Student Member, IEEE, Pierluigi Mancarella, Senior Member, IEEE \\ and Joseph Mutale, Senior Member, IEEE
}

\begin{abstract}
This paper presents a comprehensive and general optimization-based home energy management controller, incorporating several classes of domestic appliances including deferrable, curtailable, thermal, and critical ones. The operations of the appliances are controlled in response to dynamic price signals to reduce the consumer's electricity bill whilst minimizing the daily volume of curtailed energy and therefore considering the user's comfort level. To avoid shifting most portion of consumer demand towards the least price intervals, which could create network issues due to loss of diversity, higher prices are applied when the consumer's demand goes beyond a power threshold level. The arising mixed integer nonlinear optimization problem is solved in an iterative manner rolling throughout the day to follow the changes in the anticipated price signals and the variations in the controller inputs while information is updated. The results from different realistic case studies show the effectiveness of the proposed controller to minimize the household's daily electricity bill while preserving comfort level as well as preventing creation of new least-price peaks.
\end{abstract}

Index Terms -- Automated demand response, Dynamic pricing, Home energy management systems, Optimization-based controller, Building energy management, Demand Response.

\section{INTRODUCTION}

$\mathrm{D}$ emand Response [1], which is defined as "the changes in electric usage of end-use customers from their normal consumption patterns in response to changes in the price of electricity" [2], is considered as a viable alternative in future distribution networks to facilitate the connection of low carbon technologies, in particular at the domestic level, without the need for reinforcement. Indeed, time-differentiated pricing signals can be deployed to provide incentives for consumers to curtail/defer their electricity use, thus releasing capacity to connect more low carbon technologies [3-5].

However, in order to achieve these potential benefits from demand response programs, a certain level of automation is required to reduce both the uncertainty in the consumer response to price signals and the complexity for the consumers to react to the fluctuating daily electricity prices. This is called Automated Demand Response (ADR) [6].

The authors are with the Electrical Energy and Power Systems (EEPS) Group, School of Electrical and Electronic Engineering, The University of Manchester, Manchester M13 9PL, UK. (email: sereen.althaher@postgrad.manchester.ac.uk, p.mancarella@manchester.ac.uk, j.mutale@manchester.ac.uk).
The design of domestic ADR is combined with key challenges that have not yet been adequately addressed in the literature. For example, the centralized or semi-centralized control schemes in [7-8] to find the best scheduling for home appliances in multiple dwellings could be implemented in relatively small microgrids. However, their application in the context of large distribution networks would prove challenging. Therefore, more advanced decentralized controller are needed at the residential premises [4]. On the other hand, although optimisation-based ADR approaches have been developed in [7-19] to minimize electricity payments, the relevant formulations do not provide comprehensive and generic modeling for all home appliances categories. More specifically, the models in $[10-13,18]$ consider only deferrable appliances while the formulation in [9] is limited to thermal appliances. A new group of appliances whose operation is non-interruptible, e.g., washing machines, is modeled in $[14,17]$. However, these models are limited to individual cycles and additional constraints would be needed to avoid overlapping between cycles. Advanced models are proposed in $[7,15-16]$ to control all the deferrable and thermal in addition to non-interruptible appliances. However, none of the aforementioned studies except [17-19] consider critical (non-controllable) appliances. In contrast, modeling of these appliances is crucial to provide adequate assessment of the potential benefits of ADR for both consumers (for bill reduction) and utilities (for peak shaving).

Despite its potential benefits, ADR may also have negative impact on load diversity [20] which might potentially result in creating new peaks at least price intervals $[7,9,14]$ and overloaded network component. In this respect, although minimization of hourly demand or of the peak-to-average demand ratio as in [17-18] may reduce the peak demand, this may not necessarily be below a certain power threshold that could create congestion and voltage issues (i.e., power threshold is not explicitly modeled); in addition, this control design approach is not simultaneously applied with minimization of energy cost. Previous studies in $[8,15-16,21$ 23] cope with this challenge by limiting the demand at each time interval below a pre-determined power threshold. However, this may lead to frequent disconnections thus breaching consumer satisfaction and consequently discouraging the participation in ADR programs. Similarly, a 
flexible pricing approach is applied in [10-12, 19] using inclining block rate, but the use of integral energy thresholds rather than power ones renders the problem inadequate to address network constraints issues.

Curtailable appliances could provide further flexibility to manage demand below the needed power threshold. However, although Direct Load Control (DLC) models [22] are plentiful, none of the ADR-optimization based approaches available in the literature provide the relevant formulation required to avoid unnecessary curtailment. In fact, in order to boost ADR business cases it is a key to consider consumer satisfaction (e.g., volume of curtailed energy) in addition to minimizing electricity payments while preserving consumption diversity. In this context, a multi-objective optimization problem is proposed in $[7,9,11-12,14-16]$ to maximize consumer satisfaction and minimize electricity bills. However, such trade-off problems may not be practically applicable since it is difficult for consumers to provide the cost for breaching their preferences in a reasonable way. Indeed, the most likely known information is the consumer's willingness to pay.

Another key challenge is to provide solutions for potential changes in the controller inputs during the day, in particular the price signal. None of the previous studies mentioned above except $[7,10,19]$ cope with the uncertainty challenge.

In this work, a general and comprehensive formulation for an optimization-based ADR controller to be implemented in a home energy management system is proposed to optimally coordinate the operation for different types of domestic appliances in response to dynamic electricity prices. The model considers the classifications of home appliances proposed in the previous studies, namely, deferrable, noninterruptible (with multi-cycle operations) and thermal (considering the relevant thermodynamic equations) appliances in addition to critical and fully curtailable ones. The proposed ADR aims to reduce the consumer's electricity bill below a desired level, whilst increasing their comfort.

The main original contributions of this paper, bridging the gaps relative to previous work, can be summarized as follows: 1. Scheduling of appliances according to both market price signal and price penalties associated to violations of power thresholds aimed at preserving network consumption diversity (i.e., to avoid creating peaks at least price intervals) without compromising consumer's comfort (in particular in terms of volume of energy curtailment).

2. Inclusion of overlaying price signals and general formulation of the relevant response model to limit the building's aggregated power consumption. In this respect, the formulations in prior works [7-9, 13-16] can be seen as sub-cases of the models introduced in this paper (by simply relaxing some of the constraints presented).

3. Modeling of fully curtailable appliances and inclusion of consumer's comfort in the optimization problem without the need for defining the costs of breaching consumer's preference (as proposed in most literature). The optimization aims to increase the consumer comfort in an iterative manner up to a level that minimizes the electricity bill below a specified one.
4. Rolling operation of the controller throughout the day to follow the changes in the anticipated controller inputs, aimed at preserving operational constraints within limits as well as achieving the controller objectives.

The corresponding mixed integer non-linear problem (MINLP) is implemented in the AIMMS high-level optimization environment [24] (see Section III for details).

The rest of this paper is structured as follows; Section II presents the main elements of the ADR controller. The relevant formulations and algorithms are given in Section III. Case studies to validate the proposed models are presented in Section IV. Finally the conclusions are drawn in Section V.

\section{ELEMENTS OF AUTOMATED DEMAND RESPONSE}

To clarify the proposed control scheme to schedule home appliances during the course of a day, the main ADR elements and an overview of the controller decision making algorithm are presented in the following sections.

\section{A. Home Appliances Classifications}

Home appliances are classified into five categories, according to the appliance type and the consumer preference:

1. Deferrable appliances include all appliances whose starting time can be shifted across the day in response to price variations, whilst achieving the required energy use within a single day. These appliances can also be broken into two technology groups, non-flexible and flexible:

- Non-flexible deferrable appliances include all appliances that have to follow a predefined power profile during the operation cycle and cannot be interrupted. For example, laundry appliances such as washing machines have to complete a 120-minute operation cycle during rinse mode.

- Flexible deferrable appliances include deferrable appliances whose power profile can be managed during the operation, either by interrupting it or reducing its power usage. For instance, the charging rate of Plug in Electrical Vehicle (PEV) can be flexibly controlled.

2. Thermal appliances: The power consumption can be controlled to maintain the temperature within the preferred deadband, for example space heating. In this paper, the ADR model considers the thermodynamic and the intertemporal characteristics for this type of appliances.

3. Curtailable appliances: This type includes those appliances that can be switched off without the need to turn them on later. The consumer can assign the priorities to operate those appliances.

4. Critical appliances: The operations of this type of appliances are uncontrolled and have to be preserved without intervention.

\section{B. Controller Inputs}

The controller receives as inputs the forecasted outdoor temperatures and price signals for the upcoming 24-h period, as well as corresponding updates during the day together with the user settings. In addition, at each time step the price is associated with a power threshold to preserve the diversity among the consumers and thus avoids potential creation of network operational problems such as overloads which could 
result from shifting significant portions of the demand towards the least price intervals. When the dwelling demand exceeds the threshold, the corresponding price will be increased. The controller inputs are updated several times during the day; for example, the price signal received from the utility is updated every three hours. Therefore, the controller has to re-schedule the operation of the appliances in response to the updated information in a rolling fashion.

\section{Overview of the ADR Decision-Making Algorithm}

The proposed ADR aims to find the best scheduling of the controllable appliances across a finite time horizon of a single day to minimize the daily bill below the consumer willingness to pay whilst achieving maximum satisfaction level. In this work, the consumer's satisfaction level is represented by the percentage volume of the daily energy curtailment relative to the potential energy consumption of the curtailable appliances. This satisfaction level, formulated as a constraint in the optimization problem to limit the volume of energy curtailment, it is assessed iteratively by the ADR controller to achieve a desired electricity payment at the end of the day. More specifically, at the first iteration the satisfaction level is initially set to unity (i.e., no curtailment is allowed) and the corresponding energy payment is found. If this is higher than the pre-defined one, then a new iteration is performed by increasing the allowable volume of curtailment e.g., by $25 \%$. This process continues until the maximum desired payment is achieved or the curtailable appliances are all switched off (see Algorithm 1 in Section III.E for details). Accordingly; the consumer's willingness to pay enables identifying bounds on the maximum satisfaction level, consequently making it easier for the consumer to select the best alternative rather than defining the cost of breaching each consumer's comfort. In this respect, the optimization problem is run iteratively as shown in Fig. 1 by reducing progressively the normalized satisfaction level until the bill falls below the desired payment.

However, the capability of ADR to achieve the desired payment depends on the controller inputs (in particular, market price signals and controllable appliances). Therefore, even adopting a zero satisfaction level (e.g., switching off all curtailable appliances), the minimum bill might not go below the desired payment. Therefore, in practice and based on the market price signals and the other controller inputs, the controller may provide the consumer with a set of electricity payment and the corresponding satisfaction levels. The user can then select the best alternative.

To follow the changes in the controller inputs during the day (intraday), the controller is triggered repetitively to reschedule the operation of the appliances in response to the update in the utility price signal, outdoor temperature and consumer preferences. This improves energy bill reduction, user's satisfaction, and preserving operational constraints within limits (e.g., indoor temperatures).

\section{Modeling Of Automated Demand Response}

This section presents the modeling of ADR elements to schedule the operation of home appliances across a horizon of

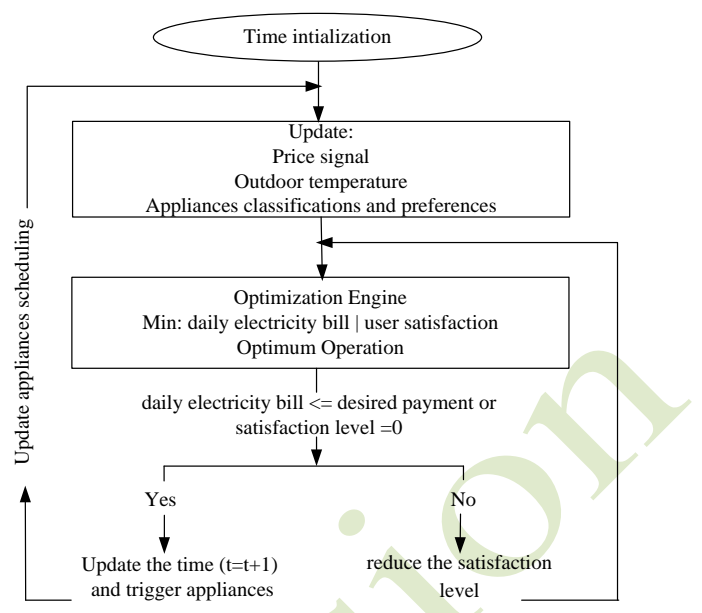

Fig. 1 Decision-making algorithm of automated demand response.

a single day, which is denoted by $\boldsymbol{K}$ (indexed by $k$ ) and it is segmented into Nequal time slots.

\section{A. Domestic Appliances Modeling}

1) Non-Flexible deferrable appliances

Each non-flexible appliance $d \in \boldsymbol{D}$ has to complete $M$ cycles of sequential operations within a day, where each operation $o \in O$ has to follow a predetermined power profile. Therefore, the optimization engine aims to find the best starting time for each appliance operation by allocating the value 1 to the corresponding time slot in the binary variable $x_{k, o}^{d}$ and according to the following set of constraints. The starting time for each operation should not deviate from the consumer preference time to begin the operation $\alpha_{o}^{d}$ by more than $\Delta_{o}^{d}$ time units. Therefore, the allowance time to start each operation should be within the interval $\lambda_{o}^{d} \equiv$ $\left[\alpha_{o}^{d}-\Delta_{o}^{d}, \alpha_{o}^{d}+\Delta_{o}^{d}\right]$, and subject to (1) and (2).

$$
\begin{array}{ll}
\sum_{k \in \lambda_{o}^{d}} x_{k, o}^{d}=1 & \forall d \in \boldsymbol{D}, o \in \boldsymbol{O} \\
x_{k, o}^{d}=0, & \forall k \in \boldsymbol{K} \backslash \lambda_{o}^{d}, d \in \boldsymbol{D}, o \in \boldsymbol{O}
\end{array}
$$

All the operations should complete a full cycle $\tau_{o}^{d}$ before the end of the day (at time step $N-1$ ); therefore the last feasible time to start any operation should be before $(N-1-$ $\left.\tau_{o}^{d}\right)$. Further, the starting time for each operation should not lie within other operation cycle as given in (3), to prevent overlapping of multiple operations for the same appliance.

$$
\sum_{k \in \lambda_{o}^{d}}\left(x_{k, o}^{d}-x_{k, o-1}^{d}\right) \varphi_{k} \geq \tau_{o-1}^{d} \quad \forall d \in \boldsymbol{D}, o \in \boldsymbol{O}
$$

where $\varphi_{k}$ represents the ordinal number relative to the time set $\boldsymbol{K}$. The power profile $p_{k, o}^{d}$ is a shifted version of the predetermined profile and it is mathematically formulated as the convolution between the predetermined profile $p_{l, o}^{d}$ and the binary decision variable (starting time) $x_{k, o}^{d}$ as given in (4).

$$
p_{k, o}^{d}=\sum_{l=0}^{N-1} p_{l, o}^{d} x_{k-l+1, o}^{d} \forall k \in \boldsymbol{K}, \forall d \in \boldsymbol{D}, o \in \boldsymbol{O}
$$

where $\boldsymbol{L}$ (indexed by $l$ ) is an alias for the time horizon set $\boldsymbol{K}$. 
Although this type of appliances has been modeled in different approaches (e.g., [14, 17]), these are limited to individual cycles and additional constraints are needed to avoid overlapping between cycles, as modeled here. In fact, the formulation of the power profile and multi-operation cycles provides substantial improvement relative to [7, 15-16].

\section{2) Flexible deferrable appliances}

For flexible deferrable appliances $f \in \boldsymbol{F}$, the controller has to achieve the required energy usage $E^{f}$ (i.e., $\mathrm{kWh}$ ) within a particular time interval $\lambda^{f} \equiv\left[\alpha^{f}, \beta^{f}\right]$, which is pre-set by the consumer, for example to fully charge the PEV before the departure time $\beta^{f}$. This is accomplished through controlling the power consumption in each time interval between zero (off) and the rated power $P^{f, \text { rated }}$. Hence, the power consumption $p_{k}^{f}$ is subject to the following constraints [11]:

$$
\begin{array}{cc}
\sum_{k \in \lambda^{f}} \frac{24}{N}\left(p_{k}^{f}\right)=E^{f} \quad \forall f \in \boldsymbol{F} \\
p_{k}^{f} \leq P^{f, \text { rated }} & \forall k \in \lambda^{f}, f \in \boldsymbol{F} \\
p_{k}^{f}=0, & \forall k \in \boldsymbol{K} \backslash \lambda^{f}, f \in \boldsymbol{F}
\end{array}
$$

\section{3) Thermal appliances}

For thermal appliances indexed by $h$, the controller has to find the corresponding power consumption $p_{k}^{h}$ at each time interval to preserve the indoor temperature $T_{k}^{(i n)}$ between the user preferred temperature settings $T^{(+,-)}$during the active periods in which the consumer turns the appliance on.

The dwelling temperature (in ${ }^{\circ} \mathrm{C}$ ) $T_{k+1}^{(i n)}$ at each time step $k+1$ is calculated using the dynamic energy balance equation in (8), which relates the indoor and the outdoor temperature between two successive time steps.

$T_{k+1}^{(\text {in })}=\varepsilon T_{k}^{(\text {in })}+(1-\varepsilon)\left(T_{k}^{(o u t)} \mp \frac{C O P \cdot p_{k}^{h}}{A}\right) \forall k \in \boldsymbol{K}, h \in \boldsymbol{H}$

The thermal model is run with (+) for heating and (-) for cooling and with the following inputs: outdoor temperature $T_{k}^{(\text {out })}\left({ }^{\circ} \mathrm{C}\right)$, the inertia factor $\varepsilon$, the thermal conductivity A $\left(\mathrm{kW} /{ }^{\circ} \mathrm{C}\right)$, and the Coefficient of Performance (COP) [9]. The inertia factor depends on the time constant of the system and the adopted control period (e.g., $15 \mathrm{~min}$ ), and its value could for instance be inferred from historical data. The $C O P$ is the ratio of the heat exchanged with the indoor ambient to the required input electrical energy [25].

\section{4) Curtailable appliances}

The controller manages the power consumption $p_{k}^{i}$ for curtailable (interruptible) appliances $i \in \boldsymbol{I}$ by controlling their operational status $s_{k}^{i}$ at each time step (On/Off) as in (9).

$$
p_{k}^{i}=s_{k}^{i} P^{i, r a t e d} O c c_{k}^{i} \forall k \in K, i \in I
$$

where $O c c_{k}^{i}$ is a binary parameter that represents the preferred periods of operating the curtailable appliances, and $P^{i, r a t e d}$ is the appliance rated power. It is once again worth noting that the modeling of curtailable appliances has been widely adopted in DLC models; however none of the ADRoptimization models have developed the relevant formulation.

\section{5) Critical appliances}

The non-controllable appliances $c \in \boldsymbol{C}$ represent those appliances which need to be operated without restrictions. In this work, the daily power consumption for all critical appliances are aggregated and modeled as a lumped demand $p_{k}^{c}$, which can be predicted from historical profiles.

\section{B. Intraday Operation Constraints}

As the controller activates several times during the day to follow the input updates, new constraints are included in the model to consider implicitly the last control actions and preserve appliance constraints such as preventing interrupting the washing machine during its operation cycle. In this context, in order to prevent interrupting non-flexible deferrable appliances during their operation cycles and avoid scheduling towards time slots prior to the current triggering time $\left(k \leq k^{(r e a l)}\right)$, the binary decision variables $x_{k, o}^{d}$ for $k \leq k^{(r e a l)}$ is enforced to equal the last control sequence $\left(x_{k, o}^{d}\right)^{(0)}$ that was produced by the controller during the last call of the optimization engine as given in (10).

$$
\left(x_{k, o}^{d}\right)=\left(x_{k, o}^{d}\right)^{(0)} \quad \forall k \leq k^{(r e a l)}, d \in \boldsymbol{D}, o \in \boldsymbol{O}
$$

Similarly, for other controllable appliances the power consumptions before the current time steps $k^{(\text {real })}$ are enforced to equal the power consumption produced by the last call of the optimization engine as given in (11).

$$
p_{k}^{f, h, i}=\left(p_{k}^{f, h, i}\right)^{(0)} \forall k \leq k^{(r e a l)}, f \in \boldsymbol{F}, h \in \boldsymbol{H}, i \in \boldsymbol{I}
$$

The constraints given in (10) and (11) provide improvement relative to the predictive ADR controller in [7, 10], which neglects the past control actions in the next decision.

\section{Dynamic Price Modeling}

It is assumed that the price signal is received from the electricity market and then it is superimposed by a power threshold, which for instance reflects the available capacity (headroom) in the distribution networks [4]. As from (12), when the aggregated demand $p_{k}^{(t o t a l)}$ of controllable and noncontrollable appliances is below the threshold demand $P^{l}$, the electricity market price $a_{k}$ is applied. When demand exceeds this threshold, a higher electricity price $b_{k}$ is applied as a penalty to prevent the controller from shifting most of the demand to least market price intervals. With such modeling of the price signal the ADR problem is represented as MINLP.

$$
\Pi_{k}\left(p_{k}^{(\text {total })}\right)=\left\{\begin{array}{lll}
a_{k}: & p_{k}^{(\text {total })} & \leq P^{l} \\
b_{k}: & p_{k}^{(\text {total })} & >P^{l}
\end{array}\right\}
$$

where, the power consumptions $p_{k}^{(\text {total })}$ at each time interval for the five appliance categories is given in (13).

$$
p_{k}^{(\text {total })}=\sum_{\substack{o \in \boldsymbol{O}, d \in \boldsymbol{D}, f \in \boldsymbol{F}, h \in \boldsymbol{H}, i \in \boldsymbol{I}, \boldsymbol{c} \in C}} p_{k, o}^{d}+p_{k}^{f}+p_{k}^{h}+p_{k}^{i}+p_{k}^{c}
$$

This price formulation is completely general and includes the design in $[7,9,14]$ whose price is independent of the demand level $\left(b_{k}=a_{k}\right)$ and the studies in $[8,15-16]$ that use 
hard constraint on the total power demand. Although flexible price design is adopted in [13], only a simplified version of flexible deferrable appliances is considered. All the mentioned MILP studies can be formulated as special cases of our model.

\section{Problem Formulation}

At each time the controller is triggered, the controller inputs (including appliances classifications, outdoor temperatures, price signals, and consumer settings to operate the appliances) are updated, as shown in Fig. 2. The controller inputs include the preferred time interval to operate deferrable appliances $\lambda^{d, f}$, the type of use for non-flexible deferrable and the corresponding power profile $p_{l, o}^{d}$, the required energy for flexible deferrable appliances $E^{f}$, the preferred indoor temperature $T^{(d e s)}$ and the corresponding deadband $T^{(+/-)}$, the active periods to use the thermal and curtailable appliances $O c c_{k}^{h, i}$, and the priority $\Gamma^{i}$ to disconnect the curtailable appliances. Then, the optimization engine is applied to find the best control sequences (decision variables), which includes the starting time for non-flexible deferrable appliances $x_{k, o}^{d}$, the daily power consumption for flexible deferrable and thermal appliances $p_{k}^{f, h}$ and the operational states $s_{k}^{i}$ (on/off) for curtailable appliances, in order to minimize the electricity payment across the time horizon $\boldsymbol{K}$.

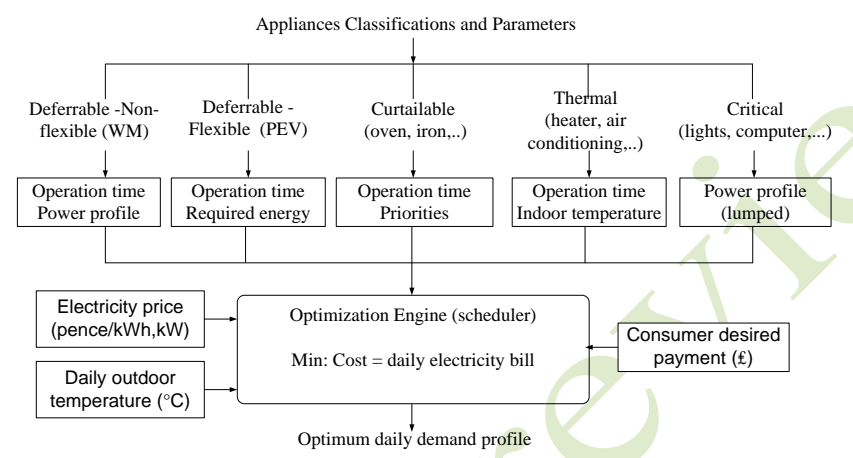

Fig. 2. Automated demand response controller architecture.

As shown in Fig. 1, the optimization is run iteratively to minimize the electricity payment below the desired electricity payment cost ${ }^{d e s}$. In order to do so, at each iteration $j$, the objective function is formulated to minimize the electricity bill across the time horizon $\boldsymbol{K}$ as given in (14).

$$
\text { Min: } \text { cost }_{j}=\sum_{k \in \boldsymbol{K}}\left(\Pi_{k}\right)\left(\frac{24}{N} * p_{k}^{(\text {total })}\right)
$$

In addition to the constraints (1-11) and the price signal in (12) in Section III-A and B, the objective function is also subject to an inequality constraint to maintain the satisfaction factor $S$ above a satisfaction level $S_{j}^{\text {des }}$.

$$
S \geq S_{j}^{\text {des }}
$$

The satisfaction level is initially set to unity (i.e., $S_{j=1}^{d e s}=1$ ) at the first iteration and it is reduced progressively by $\Delta$ until the cost falls below the desired one (i. e, cost $_{j} \leq \operatorname{cost}^{\text {des }}$ ) or when the satisfaction level reaches zero $\left(S_{j}^{\text {des }}=0\right)$. The satisfaction factor $S$ is formulated in (16) to represent the percentage of the volume of energy curtailment from curtailable appliances. Curtailment is applied according to the appliances' priority $\Gamma^{i}$ which defined as integer between 1 and the number of curtailable appliances. Those with the smallest priority values will be the first subject to curtailment.

$$
S=1-\frac{\sum_{k \in \boldsymbol{K} i \in I}\left(\rho^{\Gamma^{i}}\left(P^{i, \text { rated }} O c c_{k}^{i}-p_{k}^{i}\right)\right)}{\sum_{k \in \boldsymbol{K} i \in I}\left(\rho^{\Gamma^{i}}\left(\text { P }^{i \text { rated }}\right) O c c_{k}^{i}\right)}
$$

where $\rho$ is large constant selected as the ratio of the largest and the smallest rated power of curtailable appliances to ensure that the smallest priority appliances will be first subject to curtailment.

It is important to mention that unless such an iterative approach is applied, no curtailable appliance will operate as the cost minimization objective will push all of them off.

\section{E. Practical Implementation and Algorithms}

The proposed ADR formulation has been modeled and solved using the mathematical programming language AIMMS [24]. AIMMS's Outer Approximation Algorithm (AOA) is adopted to solve the arising MINLP problem. The AOA iteratively solves Non Linear Programming (NLP) and Mixed Integer Programming (MIP) models to find the best solution. In particular, CONOPT and CPLEX solvers are used for NLP and MIP models, respectively [26]. In order to obtain a good (feasible) solution, the AOA has also been customized by embedding a multi-start algorithm [27] that solves the NLP model for multiple initial conditions, so as to also avoid getting trapped in local solutions.

Algorithm 1 summarizes the iterative process formulated in AIMMS to find the maximum satisfaction (according to (16)) required to minimize electricity payments below the desired level. Algorithm 2 summarizes the steps and the constraints adopted to trigger the ADR at day-ahead and throughout the day (intraday) in response to changes in the controller inputs.

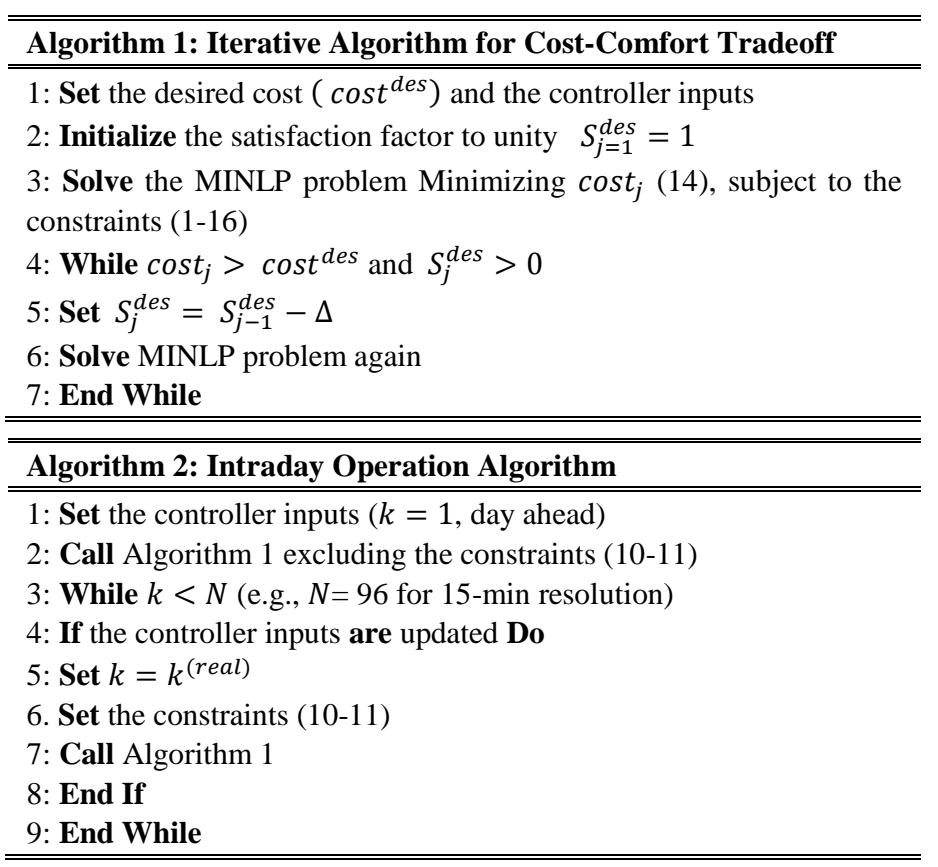




\section{CASE Study Applications}

\section{A. Description of the Case Studies}

Daily 15-min load profile for a single dwelling is developed for the coldest day in January using the model in [28]. The model generates the profiles according to historical data from UK home appliances' energy usage and depending on the number of active occupants, the season, and whether it is weekday or weekend. Then, thermal and electrical vehicle power profiles, which are not considered in [28], are generated and included in the modified model according to [29-30].

Domestic home appliances are classified into the proposed model categories. The deferrable non-flexible category includes $2 \mathrm{~kW}$ Washing Machine (WM). The user prefers to operate the WM twice a day with duty cycles of 150 and 75 minutes. The corresponding preferred starting times for each operation cycle are 18:00 and 21:00. The flexible deferrable appliance category includes $4 \mathrm{~kW}$ smart PEV charger. The corresponding charging profile should be controlled to fill the daily depleted energy of $6 \mathrm{kWh}$ during the interval from the 18:00 (arrival time) until 10:00 (departure time). Thermal appliances include a $3.5 \mathrm{~kW}$ space heater with inertia factor $\varepsilon=0.98$, thermal conductivity $A=0.45 \mathrm{~kW} /{ }^{\circ} \mathrm{C}$ and the $C O P$ set to 2.5 . The user prefers maintaining the indoor temperature close to $19^{\circ} \mathrm{C}$ within a dead-band of $2^{\circ} \mathrm{C}$ during the intervals 17:00-09:00. For the curtailable category, the consumer allows switching off the $2 \mathrm{~kW}$ oven and $3 \mathrm{~kW}$ hob appliances, considering the hob as last resort for curtailment. The consumer prefers to operate the oven and the hob concurrently during the periods 18:30-21:30, and 06:00-07:00.

Fig. 3 (a) shows the adopted price signal which represents the electricity market price and then it is superimposed by a $4 \mathrm{~kW}$ power threshold to reflect the distribution network capacity. A penalty of four times the market price at each time interval is selected to penalize the violation beyond the power threshold in particular at the minimum market price $(1.8 \mathrm{p} / \mathrm{kWh})$. This is calculated to obtain price at the minimum intervals higher than maximum $(6 \mathrm{p} / \mathrm{kWh})$. In practice, this higher price level should reflect the impact the consumer makes on network investment at each time (so-called dynamic Distribution Use of System Charge [4]). The uncontrolled load profile is plotted in Fig. 3 (b). It can be seen that the peak demand is $16 \mathrm{~kW}$, which occurs at $18: 30$ when the PEV, the thermal unit and the WM are operating concurrently. The load exceeds the power threshold for $35 \%$ of the day. The operation of domestic appliances according to the user preference without any form of control would lead to total daily energy consumption of $87 \mathrm{kWh}$ and with payment of $£ 13.5$.

In this section, the operation of the proposed controller is first demonstrated in response to the day-ahead price signal. The controller searches for the maximum satisfaction level (minimum curtailed energy) to achieve electricity payment below a desired payment level of $£ 3.5$ as a restrictive assumption to user willingness to pay. Then, the intra-day operation is investigated against changes in the controller inputs during the day. This includes the update of the price signal and the forecast of the outdoor temperature. The impact of combining the price signal with a power threshold is also

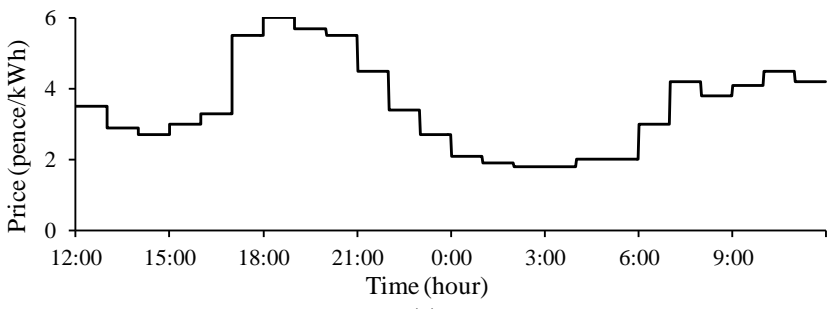

(a)

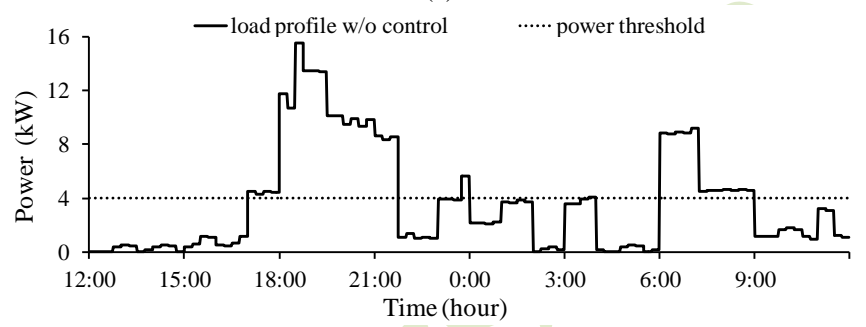

(b)

Fig. 3 (a) Hourly daily market price signal ad (b) 15-min daily load profile for a single dwelling.

presented in terms of the daily peak demand and the load factor. In addition, the minimum electricity payments and the corresponding satisfaction levels are presented to provide bounds on the feasible range of the desired payment. Finally, the proposed ADR scheme is investigated for 30 residential profiles, realistically generated according to UK statistics, to show the effectiveness of the controller in reducing electricity payment and peak demand for various inputs. The price signal in Fig. 3 (a) is adopted for all the different profiles.

\section{B. Day-Ahead Controller Operation}

In order to demonstrate the importance of adopting comprehensive modeling of home appliances, the controller will be first presented when only one category is controlled in addition to curtailable appliances. Then, all the controllable appliances are considered in the ADR to assess the impact of considering more controllable appliances on the electricity payment and the consumer satisfaction.

\section{1) Control of deferrable and curtailable appliances}

The controller has to find the best starting time of the washing machine operation cycles and the PEV charging profile. The controller reschedules the WM operations to start at 13:00 and 2:00 as shown in Fig. 4 (a). Those starting times are coincident with the maximum headroom below the threshold demand $(4 \mathrm{~kW})$. It is worth noting that if smaller waiting time is adopted, the electricity payment will increase to satisfy the constraints from (1) to (4).

On the other hand, the PEV is shifted to start charging at 21:45 and fully charging the battery before the departure time 10:00a.m as shown in Fig. 4 (b). Note, the controller utilizes the flexibility of the PEV departure time to spread the charging process over 12 hours compared to 1.5 hour in a "dumb" strategy where charging starts immediately at the arrival time using the rated charging capacity. In this case, the controller resorts to curtail all the curtailable appliances by adopting zero satisfaction level results in switching off the oven and the hob whilst achieving £6.9 daily electricity bill.

It should be highlighted that the ability to continuously 
control the PEV charging rate depends on the availability of the technology [31]. However, since the model is already formulated as MINLP, also an On-Off charging binary control strategy could be readily implemented in the controller.

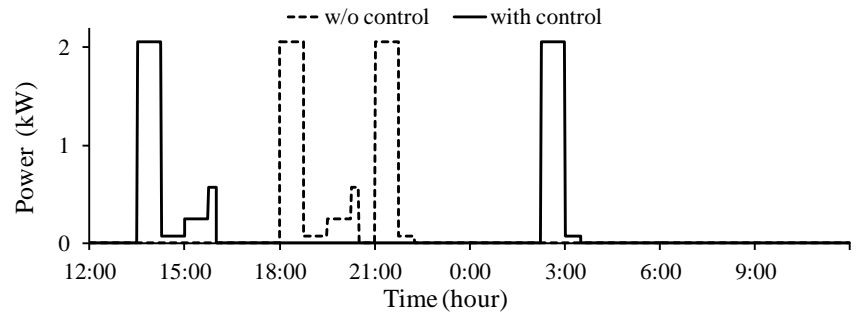

(a)

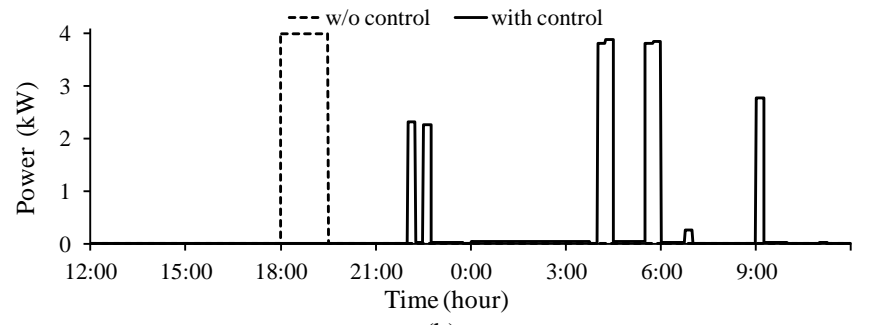

(b)

Fig. 4 Daily load profile for (a) washing machine and (b) PEV, before and after controlling deferrable and curtailable appliances.

\section{2) Control of thermal and curtailable appliances}

The power profiles of thermal appliances and the indoor temperature before and after the adoption of the proposed ADR controller are presented in Fig. 5. In the case with no control (w/o control), the use of thermal appliances is only limited to the time intervals with active occupancy. On the other hand, the ADR controller control the power usage of thermal appliances throughout the day (including with no occupancy) to minimize the electricity payment whilst keeping the indoor temperature during the occupancy intervals within the preference limits $17-21^{\circ} \mathrm{C}$. Nevertheless, the daily electricity bill can only be reduced down to $£ 4$ (still higher than desired) and again with switching off all the curtailable appliances.

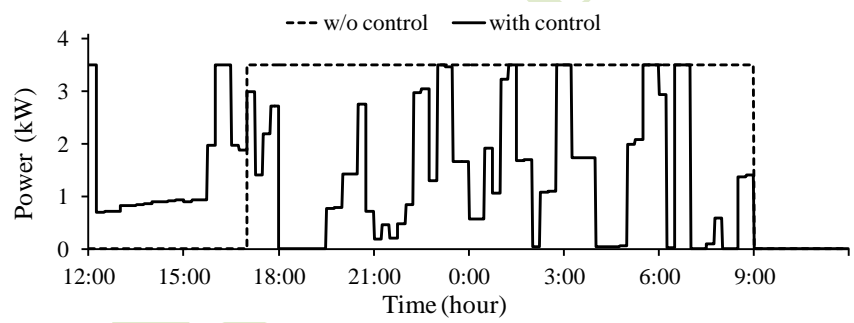

(a)

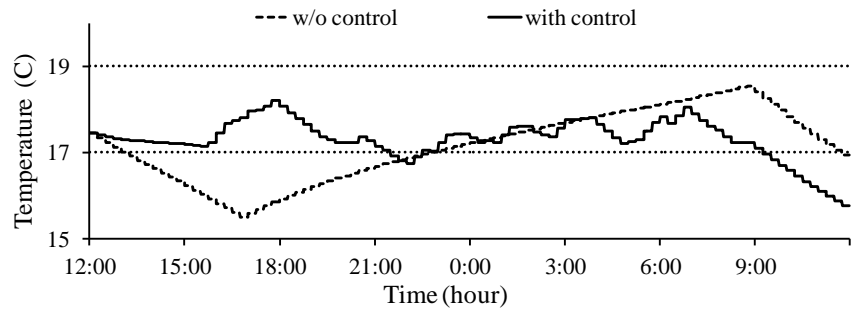

(b)

Fig. 5 (a) daily power profile and (b) indoor temperarure for the thermal appliance, without (w/o) and with control of the operation of thermal and curtailable appliances.
3) Control of deferrable, thermal and curtailable appliances (full $A D R$ )

In this case, controlling thermal appliances allows scheduling the operation of PEV and WM towards the least price intervals between 01:30-03:00 thus reducing the electricity payment. This is due to the flexibility of distributing the power usage of thermal appliances during the day as shown in Fig. 6 (a) whilst preserving the temperature constraints. The daily load profile after applying full ADR is presented in Fig. 6 (b). In the iterative process, the controller starts with unity satisfaction level to minimize electricity payment which results in payment of $₹ 7$. Then, the satisfaction is decreased gradually until the desired energy payment is below the £3.5. This is only applied at satisfaction level of 0.45 , which shows an improvement in the performance from zero satisfaction level in the previous sections. In this context, the oven and the hob are allowed to operate for $11 \%$ and $68 \%$, respectively of their occupied time intervals since the hob has the highest priority to operate. It can be seen also that for most of the day the total demand is below $4 \mathrm{~kW}$ (power threshold) due to controlling more appliance categories. However, violation exists for few time intervals when demand includes critical appliances and in the case of low outdoor temperature.

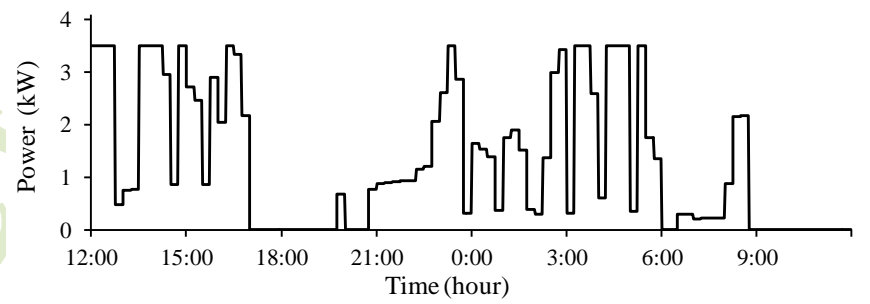

(a)

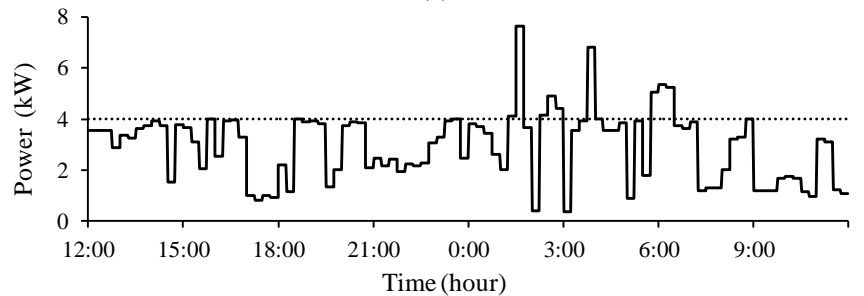

(b)

Fig. 6 Daily load profile for (a) thermal appliance, and (b) whole single dwelling after applying full ADR

\section{Intraday Controller Operation}

In this section, intra-day operation is presented to demonstrate the effectiveness of the proposed controller to cope with the updates in the price signals and the controller inputs through re-scheduling the appliances and modifying the satisfaction level to achieve the desired electricity bill at the end of the day. In this context, the price signal and the outdoor temperature are updated at 17:45 and 23:45, respectively.

\section{1) Updated price signal}

The updated forecasted price at 17:45 indicates that the price between the midnight and 6:00a.m is tripled compared to the day-ahead ones. Unless the controller re-schedules the operation, the electricity payment at the end of the day will exceed the desired payment of $£ 3.5$ by $57 \%$. Therefore, the controller is triggered to re-schedule the appliances operation. 
It can be seen in Fig. 7 (a) that the controller shifts portion of the loads towards the new least price intervals between 23:0000:00. For example, the second operation of the WM is committed to start the job earlier of three hours than the dayahead schedule at 23:00. Furthermore, the satisfaction level is reduced from 0.45 in the day-ahead to 0.25 , which leads to increase the daily energy curtailments though the curtailable appliances being turned off between 18:30-21:30.This new rescheduling preserves the electricity payment at the end of the day below the desired payment as shown in Fig. 7 (b). However, although the cost is increased during the least price intervals, the overall electricity payment at the end of the day is maintained below the desired one.

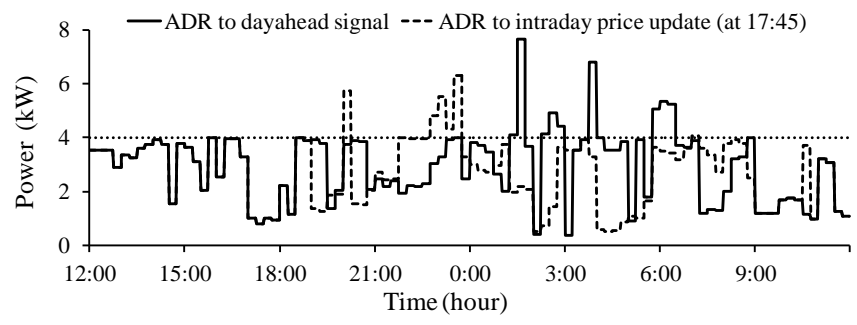

(a)

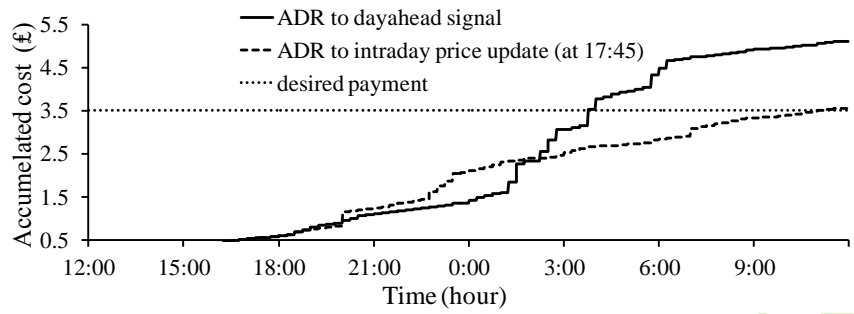

(b)

Fig. 7 (a) daily load profile and (b) accumulative cost for day ahead and intra-day sechduling for an update in the price at 17:45.

\section{2) Update outdoor temperature}

At 23:45, a new update of the outdoor temperature forecast is provided. It indicates a reduction in the forecasted temperature between midnight and 6:00a.m by $3 \mathrm{C}^{\circ}$ compared to the day-ahead forecast. Based on this, and the last scheduling carried out at 17:45, the indoor temperature would violate the minimum user preference indoor temperature of $17 \mathrm{C}^{\circ}$ during the occupancy intervals between 06:00-09:00, as shown in Fig. 8 (a). To cater for this, the controller is triggered to find new schedule for the appliances. The corresponding new load profile is presented in Fig. 8 (b). It can be also seen that the demand is increased slightly between 03:00-06:00 to preserve the indoor temperature within limits during the occupancy intervals. However, to maintain the cost below the desired one, all the curtailable appliances during this period are not allowed to operate thus the satisfaction level is reduced from 0.25 to zero.

\section{ADR Performance-Single dwelling}

This section presents the impact of combining the market price signal with a power threshold. Three metrics are used to assess the performance of the proposed ADR; the daily peak demand, the duration of violating the threshold (\% of time intervals in the day whose demand levels are above the $4 \mathrm{~kW}$ power threshold) and the load factor.

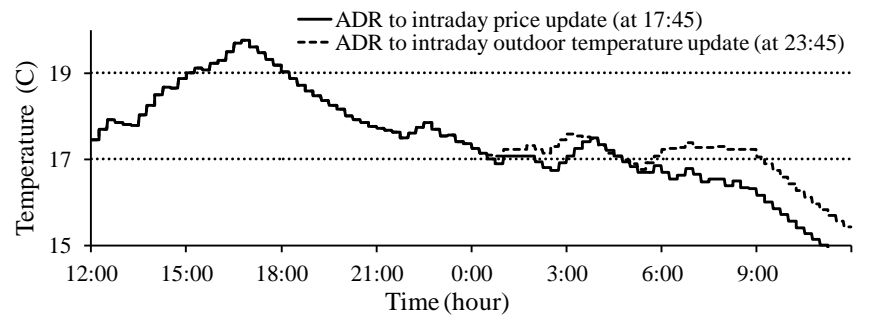

(a)

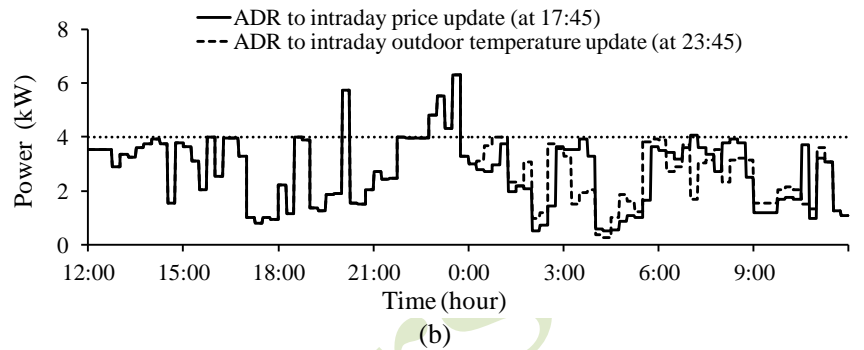

Fig. 8 (a) indoor tempreture and (b) daily load profile in response to update in the price at 17:45 and the outdoor temperature at 23:45.

The metrics are compared when threshold is not applied. Results are presented in Table I and for different satisfaction levels. From the utility perspective, the threshold power allows a dramatic reduction in the peak demand and the percentage of time intervals whose demand is higher than the power threshold. Furthermore, the load factor is dramatically improved which may lead to better utilization of the network assets. However, these potential benefits may be reduced when consumers are not concerned about the electricity payments, for example at satisfaction level of 0.75 .

Fig. 9 illustrates the relevant daily load profiles. It can be seen that adopting price signals without threshold creates new peak demand of $8.9 \mathrm{~kW}$ during the period 01:00 to 03:00a.m compared to $4 \mathrm{~kW}$ in the original profile before demand response. When the power threshold is applied, the demand is managed below $4 \mathrm{~kW}$ for most of the time intervals. However, there is need to extend this analysis to more consumers to assess the effectiveness to manage network constraints.

From the consumer perspective, the violation above the power threshold for few time intervals demonstrates the flexibility offered by the adopted price design to cater for the critical appliances and maintain the operational constraints within limits. This flexibility has not being considered in the studies $[8,15-16,21-23]$ where the total demand is limited up to the power threshold. Therefore, the home appliances will be tripped during $2 \%$ to $23 \%$ of the day which in turn may discourage the penetration of ADR.

Table I also represents the set from which the consumer can identify the desired electricity payment. In practice, the controller may provide this matrix when the controller inputs have been updated. In this case study, the minimum feasible desired electricity payment is $£ 2.3$ for the day but it will be combined with switching off all the curtailable appliances.

\section{E. Case Study-Group of 30 Dwellings}

The proposed ADR scheme is applied here to 30 daily residential profiles generated from the model in [28] for a week day in January and according to UK statistics on number of occupants per dwelling. 
TABLE I

ADR PERFORMANCE FOR SINGLE DWELLING

\begin{tabular}{c|c|c|c|c|c}
\hline $\begin{array}{c}\text { Price } \\
\text { Scheme }\end{array}$ & $\begin{array}{c}\text { Satisfaction } \\
\text { level }\end{array}$ & $\begin{array}{c}\text { Duration } \\
\text { above } \\
\text { 4kW }(\%)\end{array}$ & $\begin{array}{c}\text { Peak } \\
(\mathrm{kW})\end{array}$ & $\begin{array}{c}\text { Load } \\
\text { factor } \\
(\%)\end{array}$ & $\begin{array}{c}\text { Electricity } \\
\text { bill (£) }\end{array}$ \\
\hline \multirow{2}{*}{$\begin{array}{c}\text { Without } \\
\text { power } \\
\text { constraint }\end{array}$} & 0.00 & 21 & 8.9 & 28 & 1.74 \\
\cline { 2 - 6 } & 0.25 & 25 & 8.9 & 30 & 1.93 \\
\cline { 2 - 6 } & 0.50 & 28 & 8 & 36 & 2.18 \\
\hline \multirow{2}{*}{$\begin{array}{c}\text { With } \\
\text { power } \\
\text { constraint } \\
\text { of 4kW }\end{array}$} & 0.75 & 31 & 8 & 39 & 2.46 \\
\cline { 2 - 6 } & 0.00 & 2 & 4.5 & 56 & 2.30 \\
\hline & 0.25 & 6 & 6.3 & 43 & 2.35 \\
\hline
\end{tabular}

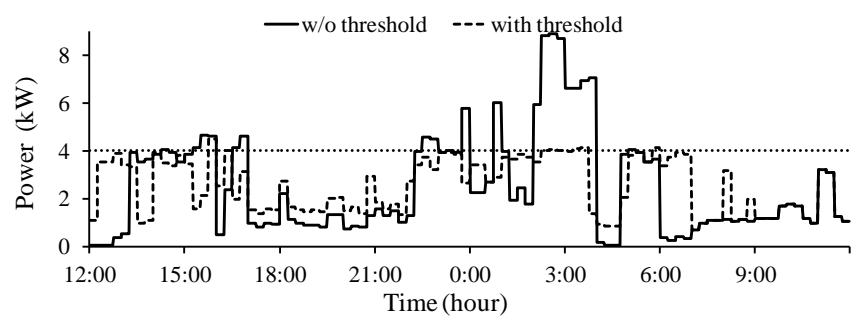

Fig. 9 Daily load profile using price signal with and without $4 \mathrm{~kW}$ power threshold for single dwilling.

EV profiles are also produced according to the UK statistics of users' driven distance (e.g., 30 miles average), arrival times (average18:00), and departure times (08:00) [2930] [32]. EV chargers of $3 \mathrm{~kW}$ and $4 \mathrm{~kW}$ are adopted and uniformly distributed among the profiles. In addition, thermal power profiles are produced according to the outdoor temperature of the coldest UK winter day in January. The preferred indoor temperature is in the range of $18^{\circ} \mathrm{C}$ and $22^{\circ} \mathrm{C}$ according to the UK statistics and with allowance of $3^{\circ} \mathrm{C}$ [33]. The ratings of thermal appliances are $3.5 \mathrm{~kW}, 4.5 \mathrm{~kW}$ and 5.5 $\mathrm{kW}$, also uniformly distributed among the profiles. The feasible waiting time for non-flexible shiftable appliances (e.g., WMs) is distributed uniformly within five hours from the starting time generated in the model. The desired satisfaction levels are also uniformly distributed from 0 to 1 .

Fig. 10 shows the benefits of the proposed ADR scheme according to the performance metrics in section IV-D.

From the results, the energy payments (Fig. 10(a)) are reduced significantly by an average of $68 \%$ for all the profiles; peak demand is also reduced below the $4 \mathrm{~kW}$ threshold in $57 \%$ of the cases (Fig. 10(b)), while the time intervals whose demand is exceeding the $4 \mathrm{~kW}$ threshold (Fig. 10(c)) is also reduced for all the profiles from an average of $25 \%$ to $4.5 \%$. Also, the load factor is improved for all the profiles from an average of $30 \%$ to $43 \%$. It can also be seen that the potential benefits from ADR are smaller for profiles whose controller inputs do not provide enough flexibility to go below the $4 \mathrm{~kW}$ threshold. For example, the first profile (profile number 0) has relatively high critical demand of $8 \mathrm{~kW}$ that cannot be reduced further (although ADR manages to reduce the peak from over $12 \mathrm{~kW}$ ). Nonetheless, ADR reduce the dwelling's energy payment from $£ 9.2$ to $£ 3.5$ and its duration of exceeding the peak from $28 \%$ to $11.5 \%$, as well as improve its load factor slightly by $4 \%$. This also shows the importance of modeling the critical appliances not to overestimate ADR benefits.

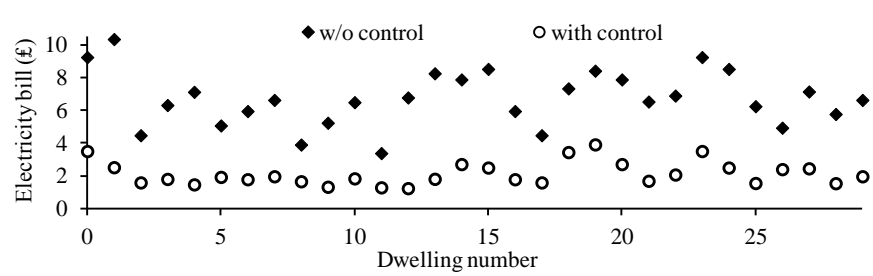

(a)

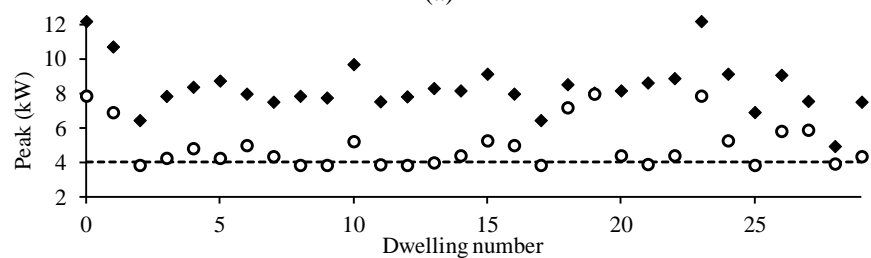

(b)

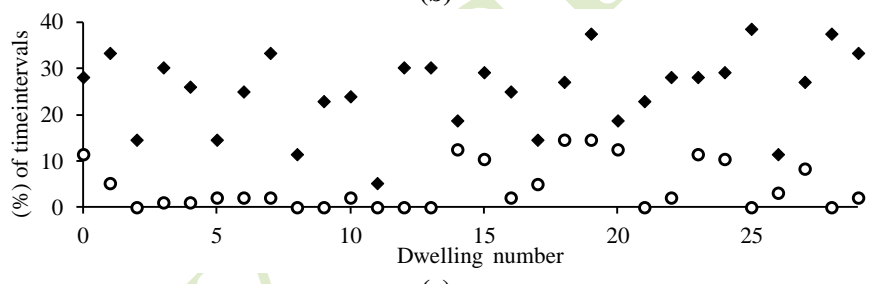

(c)

Fig. 10 Performance indicators for 30 users with and without control: (a) daily electricity bill (£) (b) peak power $(\mathrm{kW})$ and (c) \% of time intervals in the day whose demand levels are above the $4 \mathrm{~kW}$ power threshold.

\section{CONCLUSIONS}

A comprehensive optimization-based ADR controller has been proposed to optimally coordinate the operation for different types of domestic appliances to reduce the consumer's electricity bill below a predefined desired one whilst maintaining their comfort. The modeling of home appliances has been extensively developed to include all the classifications proposed in the literature. The proposed ADR scheme applied to an individual dwelling demonstrates the importance of adopting comprehensive modeling of home appliances to exploit the flexibility within each appliance category. In particular, the realistic modeling of critical (uncontrollable) appliances provides adequate assessment of ADR without overestimating its value. Furthermore, the adoption of a general and flexible price design through the application of electricity rates higher than the market price signals to penalize the violation of a given power threshold can limit the creation of a new peak at the least price intervals while still not compromising consumer's comfort. Finally, the results reveal the importance of rolling operation of the proposed controller throughout the day to follow the changes in the price signal and the outdoor temperature. This results in minimizing the electricity bill while avoiding violation of the indoor temperature preference.

The model has also been applied to 30 residential profiles. The results show the effectiveness of the controller to reduce the electricity payment and peak demand under various conditions, as well as the importance of modeling critical appliances so as not to overestimate the benefits from ADR.

Work in progress aims at assessing the implications of the proposed ADR schemes for network pricing. 


\section{REFERENCES}

[1] R. Belhomme, et al., "ADDRESS: Technical and Commercial Conceptual Architectures," The European communities seventh framework program (FP7), 2009.

[2] "Benefits of Demand Response in Electricity Markets and Recommendations for Achieving Them," US Department of Energy, 2006.

[3] M. Albadi and E. El-Saadany, "Demand Response in Electricity Markets: An Overview," in IEEE/PES General Meeting, 2007, pp. 1-8.

[4] "Network Tariff Structure for a Smart Energy System," Union of the Electricity Industry - EURELECTRIC, 2013

[5] D. Papadaskalopoulos and G. Strbac, "Decentralized Participation of Flexible Demand in Electricity Markets-Part I: Market Mechanism," IEEE Trans. on Power Systems vol. 28, no. 4, pp. 3658-3666, 2013.

[6] Department of Energy and Climate Change (DECC), "Demand Side Response in the domestic sector- a literature review of major trials," UK, 2012.

[7] C. Chen, W. Jianhui, H. Yeonsook, and S. Kishore, "MPC-Based Appliance Scheduling for Residential Building Energy Management Controller," IEEE Trans. on Smart Grid, vol. 4, no. 3, pp. 1401 - 1410, 2013

[8] L.Ying, L. Boon, M.Trayer, and L.Lingjia, "Automated Residential Demand Response: Algorithmic Implications of Pricing Models," IEEE Trans. on Smart Grid, vol. 3, no. 4, pp. 1712 - 1721, 2012.

[9] C. Christian, J. Lin, C. Wu, and C. Chuang, "Multi-Objective AirConditioning Control Considering Fuzzy Parameters Using Immune Clonal Selection Programming," IEEE Trans. on Smart Grid, vol. 3, no. 4, pp. 1603-1610, 2012.

[10] C. Xiaodao, W. Tongquan, and H. Shiyan, "Uncertainty-Aware Household Appliance Scheduling Considering Dynamic Electricity Pricing in Smart Home," IEEE Trans. on Smart Grid vol. 4, no. 2, pp. $932-941,2013$.

[11] A. Mohsenian-Rad and A. Leon-Garcia, "Optimal Residential Load Control with Price Prediction in Real-time Electricity Pricing Environments," IEEE Trans. on Smart Grid, vol. 1, no. 2, pp. 120 - 133 2010.

[12] Z. Zhuang, L. W. Cheol, S. Yoan, and S. Kyung-Bin, "An Optimal Power Scheduling Method for Demand Response in Home Energy Management System," IEEE Trans. on Smart Grid vol. 4, no. 3, pp. $1391-1400,2013$.

[13] Y.Ozturk, D.Senthilkumar, S. Kumar, and G.Lee, "An Intelligent Home Energy Management System to Improve Demand Response," IEEE Trans. on Smart Grid, vol. 4, no. 2, pp. 694 - 701, 2013.

[14] K. Tsui and S. Chan, "Demand Response Optimization for Smart Home Scheduling Under Real-Time Pricing " IEEE Trans. on Smart Grid, vol. 3, no. 4, pp. 1812 - 18212012.

[15] T.Hubert and S.Grijalva, "Modeling for Residential Electricity Optimization in Dynamic Pricing Environments," IEEE Trans. on Smart Grid, vol. 3, no. 4, pp. 2224 - 2231, 2012.

[16] M. Bozchalui, S. Hashmi, H. Hassen, C. Canizares, and K. Bhattacharya, "Optimal Operation of Residential Energy Hubs in Smart Grids," IEEE Trans. on Smart Grid, vol. 3, no. 4, pp. 1755 1766, 2012.

[17] Z. Zhu, J. Tang, S. Lambotharan, W. Chin, and Z. Fan, "An Integer Linear Programming and Game Theory Based Optimization for Demand-side Management in Smart Grid," in IEEE International Workshop on Smart Grid Communications and Networks, 2011, pp. 16.

[18] A. Mohsenian-Rad, V. Wong, J. Jatskevich, and A. Leon-Garcia, "Autonomous Demand Side Management Based on Game-Theoretic Energy Consumption Scheduling for the Future Smart Grid," IEEE Trans. on Smart Grid vol. 1, no. 3, pp. 320-331, 2010.

[19] P. Samadi, H.Mohsenian-Rad, V.W.S.Wong, and R.Schober, "Tackling the Load Uncertainty Challenges for Energy Consumption Scheduling in Smart Grid," IEEE Trans. on Smart Grid, vol. 4, no. 2, pp. 1007 1016, 2013

[20] G. Strbac, "Demand Side Management: Benefits and Challenges," Energy Policy, vol. 36, no. 12, pp. 4419-4426, 2008

[21] G. Costanzo, Z. Guchuan, M. Anjos, and G. Savard, "A System Architecture for Autonomous Demand Side Load Management in Smart Buildings," IEEE Trans. on Smart Grid, vol. 3, no. 4, pp. 2157 2165, 2012.

[22] M. Pipattanasomporn, M. Kuzlu, and S. Rahman, " An Algorithm for Intelligent Home Energy Management and Demand Response
Analysis," IEEE Trans. on Smart Grid, vol. 3, no. 4, pp. 2166 - 2173, 2012.

[23] S.Althaher and J.Mutale, "Management and control of residential energy through implementation of real time pricing and demand response," in IEEE/PES General Meeting, 2012, pp. 1-8.

[24] J. Bisschop and M. Roelofs, "AIMMS - The User's Guide," Paragon Decision Technology, 2006.

[25] P. Mancarella and G. Chicco, "Real-time demand response from energy shifting in Distributed Multi-Generation," IEEE Tran. on Smart Grid, vol. 4, no. 4, pp. 1928-1938, 2013.

[26] M. Hunting. Paragon Decision Technology BV The AIMMS Outer Approximation Algorithm for MINLP (using GMP functionality). [Online]. Available: http://www.aimms.com/aimms/download/whitepapers/gmp-aoa-aimms-whitepaper-2011.pdf

[27] "AIMMS Language Reference - Advanced Methods for Nonlinear Programs," 2012.

[28] I. Richardson and M. Thomson. Loughborough University Institutional. Domestic electricity demand model simulation example, [Online]. Available: http://hdl.handle.net/2134/5786

[29] J.Taylor, A.Maitra, M.Alexander, D.Brooks, and M.Duvall, "Evaluations of Plug-in Electric Vehicle Distribution System Impacts," in IEEE/PES General Meeting, 2010.

[30] D. Papadaskalopoulos, et al., "Decentralized Participation of Flexible Demand in Electricity Markets-Part II: Application With Electric Vehicles and Heat Pump Systems," IEEE Trans. on Power Systems, vol. 28, no. 4, pp. 3667 - 36742013 .

[31] "European Electricity Industry Views on Charging Electric Vehicles," Belgium, 2011.

[32] "Transport Statistics Bulletin, National Travel Survey," UK, 2008.

[33] M. Shipworth, et al., "Central Heating Thermostat Settings and Timing:Building Demographics," Building Research and Information, pp. $50-69,2010$.

\section{BIOGRAPHIES}

Sereen Althaher (M'11) received the B.Sc. and M.Sc. degrees in Electrical Engineering from the University of Jordan, Jordan, in 2004 and 2008, respectively. She is currently pursuing the Ph.D. degree at The University of Manchester, UK. Her research interests include demand response and pricing of distribution networks.

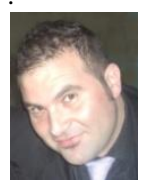

Pierluigi Mancarella (M'08, SM'14) received the Ph.D. degree in Power Systems from the Politecnico di Torino, Italy. After being a Research Associate at Imperial College London, he is currently a Reader at the University of Manchester. His research interests include multi-energy systems, business models for demand response, and network investment under uncertainty.

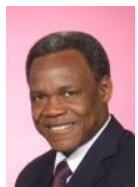

Joseph Mutale (SM'07) received the $\mathrm{PhD}$ degree in Electrical Power Systems from the University of Manchester, UK. He is currently a Senior Lecturer in the School of Electrical and Electronic Engineering at University of Manchester, UK. He is a Chartered Engineer, a Fellow of the IET. 